\title{
Practical management of NSCLC patients with long-term bevacizumab treatment: a report of four cases
}

This article was published in the following Dove Press journal:

Lung Cancer: Targets and Therapy

10 October 2013

Number of times this article has been viewed

\section{GJM Herder' \\ $\mathrm{H}$ Codrington ${ }^{2}$ \\ CD Colder ${ }^{3}$ \\ JG Aerts ${ }^{4,5}$}

'St Antonius Hospital, Nieuwegein, The Netherlands; ${ }^{2}$ Haga Hospital,

The Hague, The Netherlands;

${ }^{3}$ St Jansdal Hospital, Harderwijk,

The Netherlands; ${ }^{4}$ Amphia Hospital,

Breda; ${ }^{5}$ Erasmus MC Rotterdam, The

Netherlands
Correspondence: GJM Herder

St Antonius Ziekenhuis, Postbus 2500,

3430 EM Nieuwegein, The Netherlands

Tel +3I 0306092428

Fax $+3|088320| 449$

Email j.herder@antoniusziekenhuis.nl
Introduction: Previous research showed that the addition of bevacizumab (a monoclonal antibody against vascular endothelial growth factor [VEGF]) to chemotherapy resulted in a significant efficacy benefit in the treatment of selected patients with advanced nonsquamous non-small cell lung cancer (NSCLC). However, the occurrence and management of adverse events (AEs) during long-term maintenance treatment with bevacizumab is not well known.

Methods: This report presents a descriptive analysis, including the management of AEs, of four patients with advanced NSCLC, who received a relatively long period of bevacizumab maintenance treatment.

Results: In patient 1, a 72-year-old retired man with stage cT2N2M1b NSCLC, the only AE related to bevacizumab was a grade 1 rhinorrhea. Treatment resulted in a stable disease, with duration of response of 38 months. Patient 2 had NSCLC stage cT4N3M1b and developed a cavitation and infection after the first cycle of chemotherapy and bevacizumab, which caused a temporary decrease of her quality of life. Bevacizumab therapy resulted in a partial response, with duration of response of 15 months. A 52-year-old female (patient 3) with stage T2bN2M1a NSCLC is currently under treatment and has so far received 42 cycles of maintenance bevacizumab, with stabilized response (duration of response of 29 months) and no noteworthy AEs. The last patient is a 74-year-old male farmer with NSCLC T1N0M1, whose response has lasted for more than 3 years, with until now, no AEs.

Conclusion: Our retrospective findings of these four patients show the long-term efficacy and safety of bevacizumab treatment in a real-life setting.

Keywords: lung cancer, non-small cell lung cancer, maintenance treatment, safety management

\section{Introduction}

Lung cancer is the leading cause of cancer-related mortality in the United States and Western Europe. Around $85 \%$ of the patients with lung cancer have non-small cell lung cancer (NSCLC), of which the majority are in advanced stage (stage IV). ${ }^{1}$ According to current European guidelines, ${ }^{2}$ the first-line treatment for patients with stage IV NSCLC should take into account the histology, molecular pathology, age, performance status (PS), comorbidities, and the patient's preferences. In patients with a performance status of $0-1$, platinum-based combination chemotherapy should be offered. For patients with nonsquamous histology, pemetrexed is preferred to gemcitabine. Bevacizumab, a monoclonal antibody against vascular endothelial growth factor (VEGF), can be added to platinum-based chemotherapy in eligible patients. Previous trials and meta-analyses showed that the addition of bevacizumab 
to chemotherapy in the treatment of selected patients with advanced nonsquamous NSCLC resulted in a significant efficacy benefit. ${ }^{3-8}$ The initial bevacizumab study in NSCLC patients showed a median progression-free survival (PFS) benefit of 1.7 months and a median overall survival (OS) advantage of 2 months. ${ }^{3}$ In specifically the adenocarcinoma patients, the median OS benefit has been shown to be 3.9 months. $^{4}$

In an open-label, phase IV trial, the safety and efficacy of first-line bevacizumab combined with standard chemotherapy regimens was confirmed in clinical practice. ${ }^{9}$ The results confirmed the manageable safety profile of first-line bevacizumab in combination with various standard chemotherapy regimens for treatment of advanced nonsquamous NSCLC. ${ }^{9}$ Adverse events (AEs) of grade 3 and higher that seem to be specifically related to bevacizumab treatment are: hypertension, occurring in $\sim 7 \%$ of patients; bleeding, in $\sim 4 \%$ of patients; and proteinuria, in $\sim 3 \%$ of patients. ${ }^{3,9}$

After the induction treatment of 4-6 cycles of chemotherapy in combination with bevacizumab, treatment with bevacizumab monotherapy is continued until progression of the disease or intolerability due to toxicity. The reported median number of cycles for patients treated with bevacizumab is seven, with a range varying from one to 43 cycles (Table 1). ${ }^{3,5,9}$

The occurrence and management of long-term AEs during the maintenance treatment with bevacizumab is not well known. This report presents a descriptive analysis, including the management of AEs, of four patients with advanced NSCLC who received bevacizumab both in combination with chemotherapy and as monotherapy during the maintenance phase, for a relatively long follow-up period.

\section{Methods}

Each author had to identify the last patient from their clinical practice that received more than 20 cycles of bevacizumab treatment. Obviously, this is a highly selected cohort of patients, since the number of patients reaching $>20$ cycles is low in clinical practice. The stage, diagnosis, treatment type and duration, and the occurrence of AEs were described according to the National Cancer Institute (NCI) Common Terminology Criteria for AE (CTC-AE) 4.03. In addition, a description of the quality of life (QoL), as interpreted by the treating physician is presented. Of note; in the cases described in this paper, patient-reported QoL has not been taken into account, which could of course differ from the physician's description of QoL.

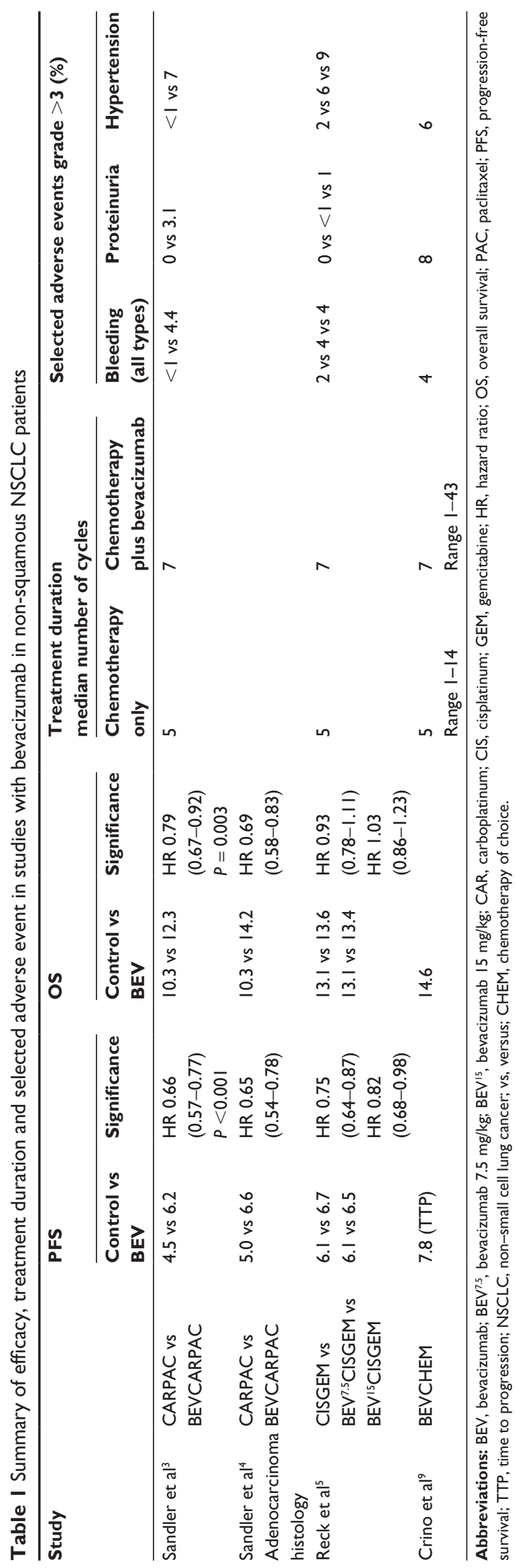




\section{Results}

\section{Patient I}

A 72-year-old retired man with a smoking history of 43-pack years presented to his primary care physician with pain in the right thorax. The pain had been present for 2 months, especially during the nights, and had been treated with tramadol and diclofenac. He had no other respiratory symptoms (no coughing, no sputum, no hemoptysis, and no dyspnea), his body weight had been stable for thirty years. At that time, he was still able to walk the stairs without problems. A computed tomography (CT) scan of the thorax and ${ }^{18}$ fludeoxyglucose positron emission tomography $\left({ }^{18} \mathrm{FDG}-\mathrm{PET}\right)$ showed stage cT2N2M1b lung carcinoma of the left lower lobe with a lytic lesion of the rib. Cytological analysis of the lesion in the main carina showed a large-cell, poorly differentiated, thyroid transcription factor (TTF)1-positive lung tumor; rib puncture confirmed metastatic disease without epidermal growth factor receptor $(E G F R)$ KRAS mutation.

Next to radiotherapy for the bone metastasis in the rib, patient 1 received palliative chemotherapy (carboplatin area under the curve [AUC] 6 and paclitaxel $200 \mathrm{mg} / \mathrm{m}^{2}$ ) plus bevacizumab $7.5 \mathrm{mg} / \mathrm{kg}$ every 3 weeks, for 52 treatment cycles. He received paclitaxel $200 \mathrm{mg} / \mathrm{m}^{2}$ for three cycles, which was then stopped because of allergic reaction during the fourth cycle; carboplatin $6 \mathrm{AUC}$ in combination with gemcitabine $1200 \mathrm{mg} / \mathrm{m}^{2}$ on days 1 and 8 was used in the fourth (and final) cycle of chemotherapy. Bevacizumab was continued as a single agent until disease progression.

AEs that occurred in this patient included grade 2 fatigue, grade 3 leucopenia, and grade 1 neuropathy, all of which developed during the chemotherapy and bevacizumab combination therapy. The only AE that developed during bevacizumab maintenance was a grade 1 rhinorrhea. In addition, the patient suffered from pain in his arm, of which the exact cause is unknown. Due to the pain in his arm, he had difficulty getting in and out of his car, which he had to do more than 100 times per day because of his work (newspaper-man). The maintenance treatment resulted in stable disease, with a duration of response of 38 months.

\section{Patient 2}

A 64-year-old housewife was referred by her primary care physician because of cough and pain during coughing. She was a smoker and had a smoking history of five packs per week for 40 years. The patient was in good condition (Eastern Cooperative Oncology Group performance status [ECOG PS] 1). Further examination revealed a lung adenocarcinoma in the right upper lobe, stage cT4N3M1b with metastases in the left supraclavicular nodes.

Patient 2 received chemotherapy carboplatin (AUC 6) and paclitaxel $200 \mathrm{mg} / \mathrm{m}^{2}$ for four cycles in combination with bevacizumab $15 \mathrm{mg} / \mathrm{kg}$ every 3 weeks for 21 cycles. After the first cycle, hospitalization was needed due to a cavitation in the primary tumor and a subsequent colonization of the cavity with bacteria, for which antibiotics were given. Due to this $\mathrm{AE}$, the next cycle of chemotherapy plus bevacizumab was delayed for 1 week, and the dose of each of the drugs was adjusted to $75 \%$ of the original dosage for the additional three cycles. Afterwards, bevacizumab $15 \mathrm{mg} / \mathrm{kg}$ was continued for 21 cycles until progression.

The cavitation and infection after the first cycle caused a temporary decrease in her QoL. She received a blood transfusion with two units of packed cells for anemia related to the chemotherapy bevacizumab treatment. Other AEs included grade 2 sensory neuropathy, probably due to paclitaxel, and hypertension and grade 1 proteinuria that was probably due to bevacizumab, all developing during the combination treatment. During the maintenance treatment, no additional toxicities were established. Due to a decrease in cough, her QoL improved. Treatment resulted in a partial response, with a duration of response of 15 months.

\section{Patient 3}

A 52-year-old female counter assistant, who had smoked two packs per day for 30 years, was referred to the chest physician because of severe chronic obstructive pulmonary disease (COPD) (stage GOLD III), with extensive centrilobular emphysema; no previous exacerbation; no inhalation medication, and a focal lesion in the right upper lobe and multiple, small nodular lesions in both lungs. Upon further examination, the diagnosis was a T2bN2M1a NSCLC of the right upper lobe (stage IV) with an ECOG PS of 0 . Analyses of EGFR and KRAS status were not possible.

Patient 3 was treated with carboplatin AUC 6, paclitaxel $200 \mathrm{mg} / \mathrm{m}^{2}$, and bevacizumab $7.5 \mathrm{mg} / \mathrm{kg}$ every 3 weeks (four cycles), resulting in a near complete response. She stopped working after the diagnosis but could cope with the disease and therapy. During the first treatment time, she was seen by a mental coach.

Maintenance treatment with bevacizumab $7.5 \mathrm{~kg} / \mathrm{kg}$ has been ongoing every 3 weeks for 42 cycles, with good tolerability and no noteworthy AEs, and with a stabilized response. Due to liver enzymes abnormalities that were caused by bile stones, the treatment was postponed two times. 
No treatment-related AEs were reported, and no concomitant or supportive therapy was needed.

Her QoL is currently good, the patient is in good condition, and is able to carry out daily work, but didn't return to her job. During the maintenance treatment, there were no side effects and no complaints about the repeating hospital visits every 3 weeks. The maintenance treatment resulted in a partial response, and the patient is still on treatment, with a duration of response to date, of 29 months.

\section{Patient 4}

A 74-year-old male farmer (smoking history: one pack per day for 30 years, no longer smoking) presented with abdominal pain, fatigue, and loss of appetite. He was referred by his general practitioner. His chest X-ray and CT-scan showed a tumor in the right upper lobe, with lymphadenopathy. An ultrasound of his liver showed abnormalities that were compatible with metastasis. A bronchoscopy and liver biopsy confirmed the diagnosis of NSCLC stage T1N0M1. EGFR/ $K R A S$ mutational analysis was not performed. His ECOG PS was 1.

The treatment plan constituted of carboplatin AUC 5, gemcitabine $1250 \mathrm{mg} / \mathrm{m}^{2}$, and bevacizumab $7.5 \mathrm{mg} / \mathrm{kg}$ every 3 weeks in four treatment cycles, with bevacizumab every 3 weeks as maintenance until progression. There were no side effects recorded. His ECOG PS improved to 0. The QoL is equally good. He has adjusted his activities in such manner that it will not interfere with his therapy schedule. A partial response was achieved without experiencing any AEs and with to date, a duration of response of 36 months.

\section{Discussion}

Various clinical trials have proved the efficacy of adding bevacizumab to platinum-based chemotherapy in the treatment of patients with nonsquamous NSCLC, as summarized in Table 1. The median overall survival times for patients with NSCLC treated with platinum-based chemotherapy vary from 8-10 months, as reported in previous clinical trials. ${ }^{10,11}$ The median number of cycles reported in clinical trials for patients treated with bevacizumab is seven, with a range of one to 43 cycles. $^{9}$ Also, in clinical practice, it has been observed that some NSCLC patients treated with a combination of platinum-based chemotherapy and bevacizumab are treated for a considerably longer period of time (up to 39 months). Four such cases were described in this report.

The AEs occurring in patients treated for four to six cycles with platinum-based chemotherapy and bevacizumab are generally well known and include bleeding, particularly of the nose; proteinuria; and hypertension. ${ }^{9}$ An investigation was specifically conducted to indicate the risk factors for bleeding events, since lung bleeding, in particular, can be fatal; ${ }^{12}$ this investigation showed that the only risk factor that was associated with an increased risk of bleeding was cavitation at baseline.

Treatment of patients with NSCLC in the maintenance phase is rather new, and there is not much known about the long-term effects of maintenance treatment on the occurrence of AEs. A recent report described a retrospective analysis of NSCLC patients during the maintenance phase of study E4599 and observed grade 3 or 4 hematological toxicities in less than $1 \%$; no grade 3 or 4 nausea, vomiting, or diarrhea; and no grade 5 toxicities. ${ }^{13}$ Most experience of bevacizumab maintenance treatment and toxicities has been with bevacizumab-treated patients with colorectal cancer. ${ }^{14-16}$ A recent report describes the treatment of bevacizumab in combination with various chemotherapy schemes for 1550 patients with metastatic colorectal cancer. In this report, it was also shown that bevacizumab was tolerable during the maintenance phase. This study reported a median treatment duration of bevacizumab of 6.7 months, with a range of up to 43 months. ${ }^{14}$ In colorectal cancer, it has also been shown, through patient-reported outcomes questionnaires, that bevacizumab does not have a negative impact on quality of life. ${ }^{16}$

In clinical practice, questions arise as to what AEs to expect during the maintenance phase of bevacizumab treatment and how to practically manage them. The four cases described in this report suggest that the occurrence of AEs in the maintenance phase can be mild or even almost absent, which is also in line with a retrospective analysis of study E4599. ${ }^{13}$ Obvious examples are patients 1 and 4 described in this report who received bevacizumab maintenance therapy for over 3 years in the absence of AEs. Also particularly interesting, is the description of patient 2 , in whom cavitation occurred upon treatment with bevacizumab. As described above, only cavitation at baseline is a risk factor for bleeding. Cavitation upon treatment with bevacizumab has been described previously. ${ }^{17}$ In a multidisciplinary discussion amongst clinicians from various hospitals, it was decided to continue treatment of this patient; despite the cavitation formation, this patient underwent 21 cycles and continued with her daily activities, and bleeding didn't occur.

Obviously this paper has a selection bias, as only patients who received long-term treatment were included. Patients who experienced adverse events causing treatment discontinuation were not included. The aim of the present patient 
presentation was to describe that long-term treatment can be possible.

Optimally, we should be able to use a biomarker to select "up front" the patients that will benefit from bevacizumab treatment for a long-term period. Continuing efforts are ongoing to define a biomarker, which is described recently. ${ }^{18}$

Our present retrospective findings showed the long-term efficacy and safety of bevacizumab treatment, in a selected cohort of patients that responded well to bevacizumab treatment, in a real-life setting.

\section{Acknowledgments Source of support}

Support for third-party writing assistance for this manuscript, furnished by Daniël Dresden, MD, MSc, was provided by Roche The Netherlands BV.

\section{Author contributions}

All authors contributed equally in preparation of this manuscript. GJM Herder, H Codrington, CD Colder and JG Aerts drafted the manuscript, and all authors approved the final version.

\section{Disclosure}

The authors report no conflicts of interest in this work.

\section{References}

1. Jemal A, Bray F, Center MM, et al. Global cancer statistics. CA Cancer J Clin. 2011;61(12):134.

2. Peters S, Adjei AA, Gridelli C, Reck M, Kerr K, Felip E; ESMO Guidelines Working Group. Metastatic non-small-cell lung cancer (NSCLC): ESMO Clinical Practice Guidelines for diagnosis, treatment and follow-up. Ann Oncol. 2012;23 Suppl 7:vii56-vii64.

3. Sandler A, Gray R, Perry MC, et al. Paclitaxel-carboplatin alone or with bevacizumab for non-small-cell lung cancer. $N$ Engl J Med. 2006;355: 2542-2550.

4. Sandler A, Yi J, Dahlberg S, et al. Treatment outcomes by tumor histology in Eastern Cooperative Group Study E4599 of bevacizumab with paclitaxel/carboplatin for advanced non-small cell lung cancer. JThorac Oncol. 2010;5(9):1416-1423.

5. Reck M, von Pawel J, Zatloukal P, et al. Phase III trial of cisplatin plus gemcitabine with either placebo or bevacizumab as first-line therapy for nonsquamous non-small-cell lung cancer: AVAil. J Clin Oncol. 2009;27(8):1227-1234.
6. Reck M, von Pawel J, Zatloukal P, et al; BO17704 Study Group. Overall survival with cisplatin-gemcitabine and bevacizumab or placebo as first-line therapy for nonsquamous non-small-cell lung cancer: results from a randomised phase III trial (AVAiL). Ann Oncol. 2010;21(9): 1804-1809.

7. Lima AB, Macedo LT, Sasse AD. Addition of bevacizumab to chemotherapy in advanced non-small cell lung cancer: a systematic review and meta-analysis. PLoS One. 2011;6(8):e22681.

8. Soria JC, Mauguen A, Reck M, et al; Meta-Analysis of Bevacizumab in Advanced NSCLC Collaborative Group. Systematic review and meta-analysis of randomised, phase II/III trials adding bevacizumab to platinum-based chemotherapy as first-line treatment in patients with advanced non-small-cell lung cancer. Ann Oncol. 2013;24(1):20-30.

9. Crinò L, Dansin E, Garrido P, et al. Safety and efficacy of first-line bevacizumab-based therapy in advanced non-squamous non-small-cell lung cancer (SAiL, MO19390): a phase 4 study. Lancet Oncol. 2010;11(8):733-740.

10. Schiller JH, Harrington D, Belani CP, et al; Eastern Cooperative Oncology Group. Comparison of four chemotherapy regimens for advanced non-small-cell lung cancer. $N$ Engl J Med. 2002;346(2):92-98.

11. Scagliotti GV, Parikh P, von Pawel J, et al. Phase III study comparing cisplatin plus gemcitabine with cisplatin plus pemetrexed in chemotherapy-naive patients with advanced-stage non-small-cell lung cancer. J Clin Oncol. 2008;26(21):3543-3551.

12. Sandler AB, Schiller JH, Gray R, et al. Retrospective evaluation of the clinical and radiographic risk factors associated with severe pulmonary hemorrhage in first-line advanced, unresectable non-small-cell lung cancer treated with Carboplatin and Paclitaxel plus bevacizumab. J Clin Oncol. 2009;27:1405-1412.

13. Lopez-Chavez A, Young T, Fages S, et al. Bevacizumab maintenance in patients with advanced non-small-cell lung cancer, clinical patterns, and outcomes in the Eastern Cooperative Oncology Group 4599 Study: results of an exploratory analysis. J Thorac Oncol. 2012;7(11): 1707-1712.

14. Bendell JC, Bekaii-Saab TS, Cohn AL, et al. Treatment patterns and clinical outcomes in patients with metastatic colorectal cancer initially treated with FOLFOX-bevacizumab or FOLFIRI-bevacizumab: results from ARIES, a bevacizumab observational cohort study. Oncologist. 2012;17(12):1486-1495.

15. Hurwitz HI, Honeycutt W, Haley S, Favaro J. Long-term treatment with bevacizumab for patients with metastatic colorectal cancer: case report. Clin Colorectal Cancer. 2006;6(1):66-69.

16. Tebbutt NC, Wilson K, Gebski VJ, et al. Capecitabine, bevacizumab, and mitomycin in first-line treatment of metastatic colorectal cancer: results of the Australasian Gastrointestinal Trials Group Randomized Phase III MAX Study. J Clin Oncol. 2010;28(19):3191-3198.

17. Nishino M, Cryer SK, Okajima Y, et al. Tumoral cavitation in patients with non-small-cell lung cancer treated with antiangiogenic therapy using bevacizumab. Cancer Imaging. 2012;12:225-235.

18. Lambrechts D, Claes B, Delmar P, et al. VEGF pathway genetic variants as biomarkers of treatment outcome with bevacizumab: an analysis of data from the AViTA and AVOREN randomised trials. Lancet Oncol. 2012;13(7):724-733.
Lung Cancer: Targets and Therapy

\section{Publish your work in this journal}

Lung Cancer: Targets and Therapy is an international, peer-reviewed, open access journal focusing on lung cancer research, identification of therapeutic targets and the optimal use of preventative and integrated treatment interventions to achieve improved outcomes, enhanced survival and quality of life for the cancer patient. Specific topics covered in the jurnalinclude: Epidemiology, detection and screen Cellular research and biomarkers; Identification of biotargets and agents with novel

\section{Dovepress}

mechanisms of action; Optimal clinical use of existing anticancer agents, including combination therapies; Radiation and surgery; Palliative care; Patient adherence, quality of life, satisfaction; Health economic evaluations. The manuscript management system is completely online and includes a very quick and fair peer-review system. Visit http://www.dovepress.com/testimonials.php to read real quotes from system. Visit http:/
published authors. 